\section{Cardio-oncology: a new discipline in medicine to lead us into truly integrative care}
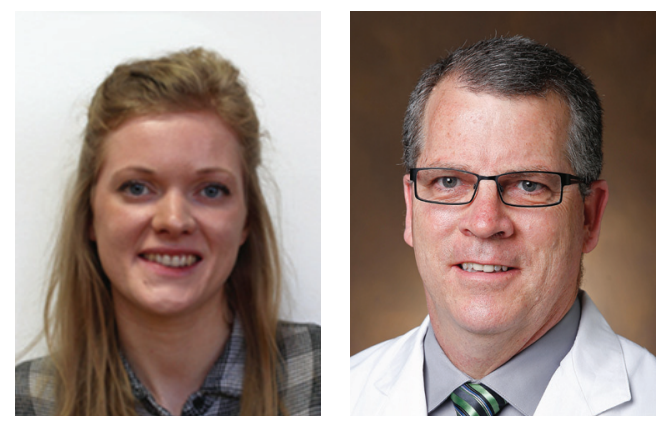

\author{
“...with an astonishing \\ improvement in cancer \\ survival rates over the past \\ two decades the \\ cardiovascular effects of \\ cancer therapies are \\ becoming increasingly \\ relevant.”
}

Ellen Clarke1 \& Daniel Lenihan ${ }^{*, 2}$

This themed issue of Future Cardiology, guest edited by Daniel Lenihan and Steven Lipshultz, fully introduces the myriad of important clinical practice issues and emerging science that encompasses the newly identified discipline of cardiooncology, primarily from a cardiovascular perspective. Many existing and emerging cancer therapies have been linked with adverse cardiovascular events, and with an astonishing improvement in cancer survival rates over the past two decades the cardiovascular effects of cancer therapies are becoming increasingly relevant [1].

In the interview in this issue, Parashar discusses the emerging field of cardiooncology, highlighting both advances and challenges within the field. She focuses on the need for collaboration between cardiologists and oncologists to improve the cardiac health of cancer survivors [2]. Jahangir et al. explore the idea that as data on the relationships between lipids, statins and cancer continue to accumulate, the role of cardiovascular specialists may expand from prevention and treatment of cardiovascular disease to interventions aimed at reducing cancer incidence and mortality [3].

It is increasingly being recognized that cancer therapeutics have unintended cardiovascular toxicity [1]. Codón, Rodríguez and López-Fernández provide insight into cardiotoxicity focusing on the known mechanisms related to cardiac dysfunction and the techniques utilized in the cardiac evaluation of cancer patients [4]. More than 25 different chemotherapeutic agents that have been linked with cardiac adverse events and a number of articles within the issue focus on this topic. Storozynsky overviews the current and emerging strategies for assessment and treatment of chemotherapy-induced cardiotoxicity [5], while Piper reviews chemotherapy-related heart failure and chemotherapeutic agents [6], Fradley and Pinilla-Ibarz look at the arrhythmic complications of tyrosine kinase inhibitors [7] while Salvatorelli, Menna and Minotti focus on the management of anthracycline-induced cardiotoxicity [8]. Patients with cancer who are treated with radiation therapy clearly experience higher rates of

\section{KEYWORDS}

- advanced imaging • biomarkers

- cardio-oncology • cardiotoxicity

"There is a compelling need for improvement in our ability to accurately predict the risk and improve early detection of cardiotoxicity.” 


\section{"In this relatively new discipline, building an adequate and functional cardio-oncology program \\ is associated with significant challenges...”}

cardiac events and cardiac mortality. One area where these issues are prominent involves the current treatment of patients with esophageal cancer and this scenario is described in an editorial by Beukema, Langendijk and Muijs [9] .

There is a compelling need for improvement in our ability to accurately predict the risk and improve early detection of cardiotoxicity. In the featured opinion article, Barac importantly comments 'an advancement in this field would provide us with an opportunity to personalize therapy and improve patients' experience and outcomes' [10].

With growing evidence suggesting that early prevention and treatment can mitigate the degree of cardiotoxicity, early detection is increasingly important. Khouri et al. review the current and emerging modalities for detection of cardiotoxicity, including imaging modalities, functional capacity testing, and serum or plasma biomarkers [11]. Additionally, Collier et al. look in depth at the utilization of strain imaging to detect cancer therapeutic-related cardiac dysfunction [12].

A key and emerging theme in cardiotoxicity management is the potential of aerobic exercise training to offset the adverse effects of cancer therapeutics. In this issue, Yu and Jones [13] and Berkman and Lakoski [14] explore this idea.

With improved cancer survival rates, long-term care is becoming increasingly relevant. Mulrooney and Duprez look beyond just measuring blood pressure and left ventricular ejection fraction, in order to improve care for the growing population of cancer survivors [15]. Montoro and LópezSendón explore chronic heart failure among cancer survivors with a focus on prevention and management strategies in this field [16].
In this relatively new discipline, building an adequate and functional cardio-oncology program is associated with significant challenges, including a paucity of practice guidelines, limited research base to help define best practice and lack of reliable funding source. In this issue, Okwuosa et al. look at the process of building a cardio-oncology program in a cancer center in the USA [17].

Primary malignant cardiac tumors are rare and associated with a poor prognosis. A proper investigation and planning are crucial to achieve the best possible outcome. Lestuzzi et al. discuss the key role of the cardio-oncologist in coordinating the diagnostic approach, and in the follow-up with regards to both the possible relapses and the cardiac toxicity of the therapeutic intervention [18].

In summary, this issue of Future Cardiology gives a broad view of the field of cardio-oncology by many of the up-and-coming leaders in this new discipline. I sincerely hope that the articles presented here will encourage thoughtful discussion, engage many institutions in the development of carefully done clinical research to enhance practice and improve the cardiac care of patients with cancer.

Financial \& competing interests disclosure E Clarke is an employee of Future Medicine Ltd. The authors have no other relevant affiliations or financial involvement with any organization or entity with a financial interest in or financial conflict with the subject matter or materials discussed in the manuscript apart from those disclosed.

No writing assistance was utilized in the production of this manuscript.

10 Barac A. Improving prediction of cardiovascular complications of cancer therapy: what does the future hold? Future Cardiol. 11(4), 383-387 (2015).

11 Khouri MG, Klein MR, Velazquez EJ, Jones LW. Current and emerging modalities for detection of cardiotoxicity in cardio-oncology Future Cardiol. 11(4), 471-484 (2015).

12 Collier P, Koneru S, Tamarappoo B, Griffin B. Cardio-oncology: strain imaging to detect cancer therapeutics-related cardiac dysfunction - are we there yet? Future Cardiol. 11(4), 401-405 (2015).

13 Yu AF, Jones LW. Modulation of cardiovascular toxicity in Hodgkin lymphoma: potential role and mechanisms of aerobic training. Future Cardiol. 11(4), 441-452 (2015). 
14 Berkman AM, Lakoski SG. The potential of aerobic exercise training in mitigating cardiovascular side effects of cancer therapy. Future Cardiol. 11(4), 407-411 (2015).

15 Mulrooney DA, Duprez D. Caring for cancer survivors - more than just checking the blood pressure and measuring the ejection fraction. Future Cardiol. 11(4), 371-375 (2015).
16 Montoro N, López-Sendón JL. Heart failure years after cancer treatment. Future Cardiol. 11(4), 433-440 (2015).

17 Okwuosa TM, Akhter N, Williams KA, DeCara JM. Building a cardio-oncology program in a small to medium-sized, non-primary cancer center, academic hospital in the United States: challenges and pitfalls. Future Cardiol. 11(4), 413-420 (2015).

18 Lestuzzi C, De Paoli A, Baresic T, Miolo G, Buonadonna A. Malignant cardiac tumors; diagnosis and treatment. Future Cardiol. 11(4), 485-500 (2015). 